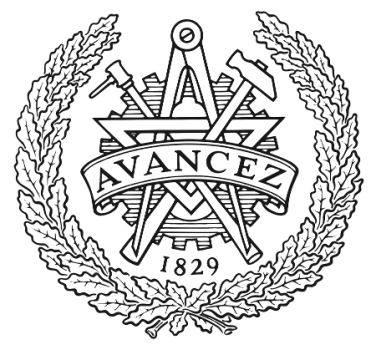

CHALMERS

UNIVERSITY OF TECHNOLOGY

\title{
Regenerator Allocation in Nonlinear Elastic Optical Networks With Random Data Rates
}

Downloaded from: https://research.chalmers.se, 2023-04-26 11:27 UTC

Citation for the original published paper (version of record):

Yan, L., Xu, Y., Brandt Pearce, M. et al (2018). Regenerator Allocation in Nonlinear Elastic Optical Networks With Random Data Rates. 2018 OPTICAL FIBER COMMUNICATIONS CONFERENCE AND EXPOSITION (OFC). http://dx.doi.org/10.1364/OFC.2018.Th2A.47

N.B. When citing this work, cite the original published paper. 


\title{
Regenerator Allocation in Nonlinear Elastic Optical Networks With Random Data Rates
}

\author{
Li Yan ${ }^{(1)}$, Yuxin Xu ${ }^{(2)}$, Maïté Brandt-Pearce ${ }^{(2)}$, Nishan Dharmaweera ${ }^{(1)}$, and \\ Erik Agrell ${ }^{(1)}$ \\ ${ }^{(1)}$ Chalmers University of Technology, Gothenburg SE-41296, Sweden \\ (2) University of Virginia, Charlottesville 22904, Virginia, USA \\ lyaa@chalmers.se
}

\begin{abstract}
We optimize the regenerator allocation in nonlinear elastic networks whose traffic demands have random data rates. Compared with previous regenerator allocation algorithms, our method achieves the same blocking probability with $11 \%$ fewer regenerator sites.
\end{abstract}

OCIS codes: (060.4256) Networks, network optimization, (060.4510) Optical communications

\section{Introduction}

The advent of colorless directionless reconfigurable optical add/drop multiplexers can support dynamic operation and optimization of elastic optical networks (EONs) [1], where sparse regenerator sites can be deployed to enable fast provisioning of services [2]. To allocate regenerator sites (RSs) efficiently and guarantee the quality of transmission (QoTs), it is necessary to accurately estimate the physical layer impairments (PLIs) and the traffic conditions in the network, which are usually tackled by using transmission reach (TR) and static traffic predictions in previous studies [1,2]. Recently, the Gaussian noise (GN) model [4] has been proposed to describe the PLIs analytically. Furthermore, enabled by bandwidth-variable wavelength cross-connects and transceivers, the network operators can dynamically change the channel bandwidth according to the real-time requirements through time-dependent bandwidth variation [3], which renders the network states extremely complicated and the static traffic prediction less useful.

In this paper, we propose a new RS allocation algorithm for nonlinear EONs with variable demand data rates. The GN model and a modified statistical network assessment process (SNAP) framework is used to estimate the PLI distributions for each demand-link pair in the network, based on which a regenerator placement algorithm is proposed.

\section{Problem Statement}

The goal is to allocate a limited number of RSs such that the blocking probability (BP) due to excessive noise is minimized, whereas abundant spectrum and transponder resources are assumed such that no demand is blocked by resource shortage. The set of demands $T$ in the EON is known with shortest-distance routing to minimize the communication delay per demand. The spectrum ordering of the demands is random since the loading process of the demands is usually unknown at the planning stage of the network. The data rate of each demand $t \in T$ is a random variable modelled by a probability density function (PDF) $p_{R_{t}}\left(r_{t}\right)$. The network topology is represented as $(V, E)$, where $V$ is the set of nodes and $E$ is the set of bidirectional links with equal-length fiber spans.

\section{Proposed Algorithm}

The proposed algorithm is consist of two parts: 1) a modified SNAP framework that determines the empirical distributions of PLIs and 2) an RS allocation heuristic.

\subsection{Modified SNAP Framework}

The PLI interfering with each demand is a function of the data rates and allocated resources of all the demands in the network. Therefore, the PLI $N_{t, l}$ suffered by the demand $t \in T$ on the link $l \in P_{t}$, where $P_{t}$ denotes the ordered set of links on the route of $t$, is also a random variable. The PDF of $N_{t, l}$, denoted as $p_{N_{t, l}}(n)$, can be statistically characterized by a modified version of the SNAP framework [5].

The modified SNAP takes as input the following information: 1) the set of traffic demands $T$ and the PDF $p_{R_{t}}\left(r_{t}\right)$ for $t \in T$; 2) the policies for routing and spectrum assignments; 3) the network topology $(V, E)$; and 4) transmission and fiber parameters that are used to determine QoTs: the physical layer fiber parameters [4], the modulation format, its corresponding signal-to-noise ratio (SNR) threshold to achieve a certain pre-FEC bit-error rate requirement, and the power spectral density (PSD) assigned to each demand. 
The modified SNAP framework outputs the PLI distributions for each demand-link pair by performing a Monte Carlo analysis. During each Monte Carlo run, a progressive load of the network is carried out to simulate one possible evolution of the network by the following steps: 1) shuffle the demands randomly and generate an ordered list of demands $\mathscr{L}(T) ; 2$ ) draw a random sample of the data rate from the PDF $p_{R_{t}}\left(r_{t}\right)$ for $\left.t \in T ; 3\right)$ load demands in $T$ with the order in $\mathscr{L}(T)$ and sampled data rates; 4) calculate the PLI for each demand-link pair based on the GN model and the bandwidths and spectrum allocations.

\subsection{RS Allocation Heuristic}

Based on the PLI distributions obtained from the Section 3.1, the RS allocation heuristic that minimizes the network BP under a fixed number $F_{\max }$ of RSs is carried out by first finding a set of promising RS allocations with low BP and as few RSs as possible for each demand. Then we select one element from the promising set for each demand such that the overall RS allocation satisfies the RS number constraint and minimizes the BP.

In order to find the promising set of RS allocations, we need to first evaluate the BP of demand $t \in T$, denoted as $H_{t}(\mathbf{f})$, as a function of the RS allocation $\mathbf{f}$, where $\mathbf{f}$ is a vector of $f_{i}$ for all $i \in V$, and $f_{i}$ is a binary indicator that equals 1 if node $i$ is an RS and 0 otherwise. Suppose $\mathbf{f}$ divides the route of $t$ into a set of transparent segments Seg $(t, \mathbf{f})$. The PDF $p_{N_{t}^{s}}(n)$ of the random accumulated noise $N_{t}^{s}$ at the end of the transparent segment $s \in \operatorname{Seg}(t, \mathbf{f})$ can be calculated by the simulated noise in Section 3.1. Then the the BP $H_{t}^{s}$ on the transparent segment $s$ can be calculated by $H_{t}^{s}=$ $\int_{G / \mathrm{SNR}_{\mathrm{th}}}^{+\infty} p_{N_{t}^{s}}(n) d n$, where $G$ is the channel PSD and $\mathrm{SNR}_{\mathrm{th}}$ is the SNR threshold of the demand. Finally the overall BP based on the assumption that the BPs on different segments are independent is given by $H_{t}(\mathbf{f})=1-\Pi_{s \in \operatorname{Seg}(t, \mathbf{f})}\left(1-H_{t}^{s}\right)$.

We identify the promising set of RS allocations $\mathscr{S}_{t}$ by finding the $k$-shortest paths [6] in an auxiliary weighted graph in $\bar{D}_{t}=\left(\bar{V}_{t}, \operatorname{Seg}(t)\right)$ with the set of nodes as $\bar{V}_{t}=\{\operatorname{src}(t)$, dst $(t)\} \cup Q_{t}$, the set of links as $\operatorname{Seg}(t)$, and the weight $w_{s}$ associated with the link $s \in \bar{E}$ as

$$
w_{s}= \begin{cases}-\ln \left(1-H_{t}^{s}\right)+\alpha, & \text { if } H_{t}^{s}<1 \\ +\infty, & \text { if } H_{t}^{s}=1,\end{cases}
$$

where $Q_{t}$ is the set of nodes on the route of $t, \operatorname{Seg}(t)$ is the set of all possible segments on the route of $t, \alpha$ is the unit regenerator cost [2], and $\operatorname{src}(t)$ and $\operatorname{dst}(t)$ are the source and destination of $t$, respectively.

Based on the sets of promising RS allocations, we select the overall RS allocation that satisfies the RS number constraint and achieves the minimum BP by solving the following optimization problem

$$
\begin{aligned}
& \underset{y_{t, \kappa}, z_{i}}{\operatorname{minimize}} \sum_{t \in T} \sum_{\kappa \in \mathscr{U} t} y_{t, \kappa} H_{t}\left(\mathbf{f}^{t, \kappa}\right) \\
& \text { subject to } \sum_{\kappa \in \mathscr{U}_{t}} y_{t, \kappa}=1 \quad \forall t \in T, \\
& \sum_{t \in T} \sum_{\kappa \in \mathscr{U}_{t}} f_{i}^{t, \kappa} y_{t, \kappa} \leq \theta z_{i}, \quad \forall i \in V \\
& \sum_{i \in V} z_{i} \leq F_{\max } .
\end{aligned}
$$

$\mathscr{U}_{t}=\left\{1, \ldots,\left|\mathscr{S}_{t}\right|\right\}$ is the set of indices for elements in $\mathscr{S}_{t}$, the binary variable $y_{t, \kappa}$ indicates if the precalculated solution $\mathbf{f}^{t, \kappa} \in \mathscr{S}_{t}$ is selected into the final RS allocation, the binary variable $z_{i}$ indicates if the node $i \in V$ is chosen as an RS, $f_{i}^{t, \kappa}$ is the $i$-th element of $\mathbf{f}^{t, \kappa}$, and $\theta$ is a large enough number. The objective (2a) calculates the overall BP in the network, (2b) implies that only one RS allocation is chosen for each demand, (2c) calculates the RS allocation for each node, and (2d) is the RS number constraint.

\section{Numerical Results}

In this section, we present the simulation results for the proposed regenerator allocation heuristic and compare it with previous algorithms [1,2]. The continental US topology (CONUS) with 75 nodes and 99 bidirectional links is studied. We assume that there is one traffic demand between each node pair. The shortest distance routes and first-fit spectrum allocation scheme are used. Polarization-multiplexing quadrature phase shift keying is applied to all the traffic demands to obtain a spectral efficiency of $4 \mathrm{bits} / \mathrm{s} / \mathrm{Hz}$ and an $\mathrm{SNR}$ threshold $\mathrm{SNR}_{\mathrm{th}}=7.03$. A uniform and fixed power spectral density $G=15 \mathrm{~W} / \mathrm{THz}$ is applied to all the traffic demands. The random data rate $R_{t}, t \in T$ is assumed to follow a normal distribution, i.e., $R_{t} \sim \mathscr{N}(\mu, \sigma)$, with $\mu=200 \mathrm{Gbps}$ and $\sigma=20 \mathrm{Gbps}$. 
In the modified SNAP, $7 \times 10^{4}$ random instances of $T$ is generated to simulate the PLI distributions. The regenerator allocation heuristic is run for the RS number $F_{\max }$ in the range between 1 to 30 . To test the BP performance, the proposed and benchmark methods are tested against $3 \times 10^{4}$ instances of $T$ with random spectrum orderings in the network. The values of TR are swept from $1300 \mathrm{~km}$ to $5000 \mathrm{~km}$ by scaling the spontaneous emission factor and nonlinear parameter proportionally according to the GN model [4].

We compare the proposed algorithm with two benchmarks based on the TR model and static traffic prediction. The first benchmark is the routing and reach heuristic (RR) [1] that ranks the likelihood of being an RS for each node based on the network connectivity and TR model. We evaluate the BP performances of the rankings computed by the proposed and RR methods, respectively. The second benchmark is the greedy constrained-routing regenerator location heuristic (greedy-CRLP) [2] that minimizes the number of RSs subject to the shortest distance routing constraint. The greedy-CRLP algorithm takes the TR value as input and outputs a set of RSs. We study the BPs of the RS sets yielded by the proposed and greedy-CRLP algorithm at the same TR.

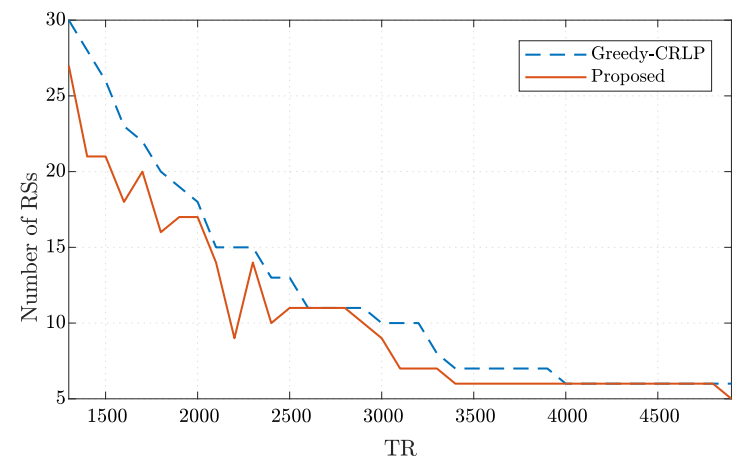

(a) The BPs of the prediction and simulation with different TRs (in $\mathrm{km}$ ).

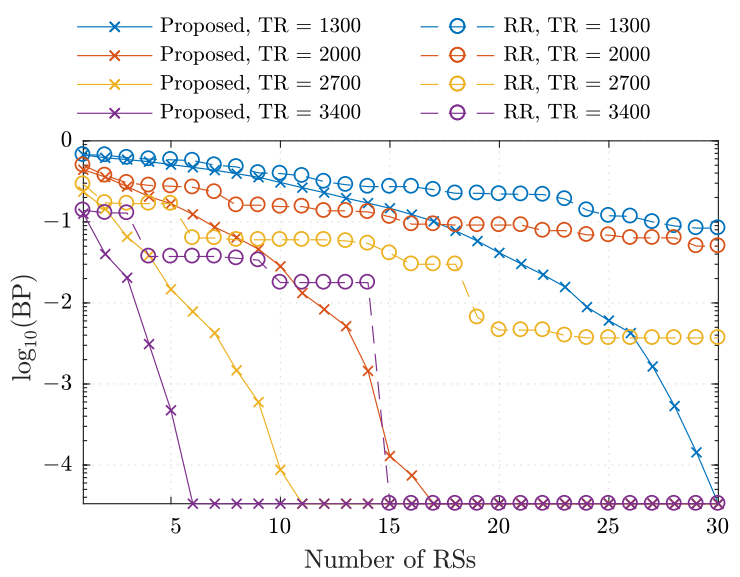

(b) Number of RSs required for the proposed and greedy-CRLP heuristics to achieve similar BP.

As is shown in Figure 1a, the proposed method uses fewer RSs to achieve the same BP compared with the greedyCRLP. On average the reduction on the number of RSs is $11 \%$. This is because the GN model and modified SNAP can accurately describe the PLI distributions in the network, which is only conservatively estimated by the TR model in the worst cases. In Figure 1b, we plot the BPs of both methods with different TRs as functions of the RS number. The BP of the proposed method has significantly lower BPs compared with the RR with an average BP gain of around two orders of magnitudes.

\section{Conclusion and Acknowledgment}

The proposed stochastic optimization framework achieves significant improvement in reducing the number of RSs and BP in the nonlinear EONs with variable demand data rates. This work is supported in part by the NSF Grant CCF-1422871, the Swedish Research Council Grant 2012-5280, and the Ericsson Research Foundation.

\section{References}

1. J. Predo, "Predeployment of regenerators for fast service provisioning in DWDM transport networks," Journal of Optical Communications and Networking, vol. 7, no. 2, pp. A190-A199, 2015.

2. B. G. Bathula et al., "Routing and regenerator planning in a carriers core ROADM networkm," Proc. Optical Fiber Communication Conference (OFC) 2017, Th4F4.

3. M. Jinno et al., "Spectrum-efficient and scalable elastic optical path network: Architecture, benefits, and enabling technologies," IEEE Communications Magazine, vol. 47, no. 11, pp. 6673, 2009.

4. P. Johannisson and E. Agrell, "Modeling of nonlinear signal distortion in fiber-optic networks," IEEE Journal of Lightwave Technology, vol. 32, no. 23, pp. 3942-3950, 2014.

5. M. Cantono et al., "Potentialities and criticalities offlexible-rate transponders in DWDM networks: A statistical approach," Journal of Optical Communications and Networking, vol. 8, no. 7, pp.A76-A85, 2016.

6. J. Yen, "Finding the $\mathrm{k}$ shortest loopless paths in a network," Management Science, vol. 17, no. 11, pp.712716, 1971. 